\title{
Control constitucional de los estados de excepción en Ecuador y Colombia: un enfoque comparado
}

\section{Constitutionality review of declarations of states of emergency: A comparative approach Ecuador and Colombia}

\section{Controlo constitucional dos estados de excepção na Equador e Colômbia: Uma abordagem comparativa}

JAIRo ANDRÉs Quitian CALDERón* 


\title{
Resumen
}

La emergencia generada por la pandemia del covid-19 constituye un nuevo capítulo en el debate del control judicial de los denominados estados de excepción. A partir de las recomendaciones efectuadas por la Organización Mundial de la Salud (OMs) para que los Estados tomaran acciones urgentes frente la identificación, el aislamiento y tratamiento de los casos confirmados, los gobiernos de Ecuador y Colombia decretaron, cada uno, el estado de excepción. El primero, por medio del Decreto Ejecutivo 1017 de 16 de marzo de 2020; el segundo, mediante el Decreto Legislativo 417 de 17 de marzo de 2020.

El presente trabajo tiene por objeto examinar el fundamento y alcance del control constitucional de estos estados de excepción en Ecuador y Colombia. Este artículo privilegia el método descriptivo, a fin de plantear una suerte de paralelismo entre los diferentes elementos de componen el control constitucional de los estados de excepción en los dos ordenamientos jurídicos.

Palabras clave: estado de excepción; control constitucional; calamidad pública.

\begin{abstract}
The emergency caused by the Covid-19 pandemic is a new chapter in the debate on judicial control of so-called states of emergency. Based on the recommendations made by the World Health Organization (wHO) for States to take urgent action for the identification, confirmation, isolation and treatment of confirmed cases, the Governments of Ecuador and Colombia decreed a state of emergency. The first, by means of Executive Decree 1017. The second, by Legislative Decree 417 of March 17, 2020.
\end{abstract}

In light of the above, the purpose of this paper is to examine the basis and scope of constitutional control of States of Emergency in Ecuador and Colombia. This article favors the descriptive method, in order to draw a kind of parallelism between the different elements that make up the constitutional control of states of emergency in these two legal systems.

Key words: State of emergency; constitutional control; public disaster.

\section{Resumo}

A emergência causada pela pandemia da CoviD-19 é um novo capítulo do debate sobre o controlo judicial dos chamados estados de emergência. Com base nas recomendações da Organização Mundial de Saúde (oms) para que os Estados tomem medidas urgentes para a identificação, confirmação, isolamento e tratamento dos casos confirmados, os 
Governos do Equador e da Colômbia decretaram, cada um deles, o estado de emergência. O primeiro, através do Decreto Executivo n. ${ }^{\circ} 1017$. O segundo, pelo Decreto Legislativo 417, de 17 de Março de 2020.

O objectivo do presente documento é examinar a base e o âmbito do controlo constitucional dos Estados de Emergência no Equador e na Colômbia. Este artigo privilegia o método descritivo, de forma a traçar uma espécie de paralelismo entre os diferentes elementos que compõem o controlo constitucional dos estados de emergência nestes dois ordenamentos jurídicos.

Palavras chave: Estado de exceção; controle constitucional; calamidade pública.

\section{Introducción}

La institución de los estados de excepción es una figura conocida por las constituciones latinoamericanas. Desde los albores de la vida independiente (Vanegas, 2011), esta institución ha sido prevista, al margen de su denominación (i.e. estado de sitio, legislación de emergencia o estados de excepción), en las constituciones de Argentina, Brasil, Bolivia, Colombia, Chile, Ecuador, El Salvador, Guatemala, Honduras, Nicaragua, Panamá, Paraguay y Venezuela (Gargarella y Roa, 2020). No hay duda acerca de la utilidad, al menos teórica, de esta institución para afrontar las más variadas situaciones de crisis no previstas en el marco de la normalidad institucional. Sin embargo, tampoco hay duda acerca de la instrumentalización que, en alguna época, supuso el establecimiento de un régimen de excepción permanente, por medio del cual varios gobiernos latinoamericanos instauraron una suerte de "excepcionalidad jurídica permanente" (García Villegas y Uprimny, 2005).

Con el propósito de conservar la institucionalidad jurídica ordinaria, la regulación constitucional, orgánica y estatutaria de los estados de excepción se fundamenta en el carácter reglado y limitado de los mismos. En efecto, mediante disposiciones constitucionales expresas, los Estados latinoamericanos han dispuesto, mutatis mutandis, un sistema de control de los estados de excepción que involucra, de un lado, instituciones políticas (controles políticos) y, del otro, instituciones jurídicas (control jurídico). El objetivo, tal como se advirtió, no es otro que regular los excesos que, en otrora época, implantaron una suerte de excepcionalidad jurídica permanente que además supuso el desconocimiento de la vigencia de los derechos fundamentales de los ciudadanos. Dicho de otra manera, el propósito de los controles políticos y jurídicos de los estados de excepción es salvaguardar la democracia y la vigencia integral de los derechos fundamentales de los ciudadanos.

Ahora bien, con ocasión de la crisis generada por la pandemia del covid-19, se ha abierto un nuevo capítulo en el debate del control judicial de los estados de excepción. Desde el 11 de marzo de 2020, la Organización Mundial de la Salud (oms) instó a los Estados a tomar acciones urgentes para la identificación, confirmación, aislamiento, monitoreo de los posibles casos y tratamiento de los casos confirmados, así como la divulgación 
de las medidas preventivas, para mitigar el contagio del nuevo virus. En los términos expuestos por la oms:

La pandemia de enfermedad por coronavirus 2019 (covid-19) está exigiendo un alto precio a personas, familias, comunidades y sociedades de todo el mundo. Las vidas cotidianas se han transformado significativamente, las economías han entrado en recesión y muchas de las redes sociales, económicas y de sanidad pública tradicionales en las que la gente confía en épocas de penuria se han visto sometidas a una presión sin precedentes. (Organización Mundial de la Salud, 2020)

De allí, que varios países hayan acudido a la institución de los Estados de Excepción para adoptar medidas extraordinarias y urgentes, con el fin de enervar o mitigar los efectos adversos generados por la pandemia del covid-19 ${ }^{1}$.

En particular, los gobiernos de Ecuador y Colombia, acogiendo las recomendaciones de la oms, decidieron decretar el estado de excepción en la totalidad de sus territorios. El primero, por medio del Decreto Ejecutivo 1017 relativo al estado de excepción por calamidad pública por la pandemia del covid-19. El segundo, mediante el Decreto Legislativo 417 de 17 de marzo de 2020, por el cual se declara un estado de emergencia económica, social y ecológica en todo el territorio nacional.

El control judicial de estos decretos corresponde, de un lado, a la Corte Constitucional del Ecuador, como máximo órgano de control, interpretación constitucional y de administración de justicia en esta materia. Por el otro, a la Corte Constitucional de Colombia, como máximo intérprete del contenido y alcance de los preceptos de la Constitución Política.

Así las cosas, el presente trabajo tiene por objeto examinar el fundamento y el alcance del control judicial de los estados de excepción en Ecuador y en Colombia. Este artículo privilegia el método descriptivo, a fin de plantear una suerte de paralelismo entre los diferentes elementos de componen el control constitucional de los estados de excepción en estos ordenamientos jurídicos. Esto supone renunciar a ciertas cuestiones problemáticas del control judicial que, aunque podrían ser abordadas desde un punto de vista analítico, escapan al objeto del presente escrito.

\section{La institución del estado de excepción en la Constitución Política del Ecuador}

De acuerdo con el artículo 164 de la Constitución Política del Ecuador (CPE), son cuatro las causas que habilitan al presidente de la República para decretar el estado de excepción

1 "Los poderes de emergencia deben usarse dentro de los parámetros estipulados por el derecho internacional de los derechos humanos, en particular por el Pacto Internacional de Derechos Civiles y Políticos (ICCPR, por sus siglas en inglés), en el que se reconoce que los Estados pueden necesitar facultades adicionales para abordar situaciones excepcionales. Dichas facultades deben conllevar plazos determinados y solo deberían ejercerse con carácter temporal, a fin de restaurar la normalidad lo más pronto posible" (ACNUDH, 2020). 
en todo el territorio nacional o en parte de él. Según la citada disposición, la declaratoria de estado de excepción procede: "en caso de agresión, conflicto armado internacional o interno, grave conmoción interna, calamidad pública o desastre natural”. Así, por ejemplo, por medio del Dictamen 2-20-EE/20, la Corte Constitucional del Ecuador consideró que tratándose de la pandemia del covid-19, la causal de calamidad pública para decretar el estado de excepción se encontraba comprobada por las autoridades de salud y los organismos internacionales, dado el número de personas fallecidas y contagiadas a nivel nacional.

Ahora bien, en los términos de la CPE, la declaratoria del estado de excepción se regirá, en todos los casos, por los principios de necesidad, proporcionalidad, legalidad, temporalidad, territorialidad y razonabilidad. Esta precisión tiene por objeto limitar las facultades extraordinarias que tiene el Ejecutivo para, de un lado, decretar el estado de excepción y, del otro, adoptar medidas en el marco de esta institución. En efecto, a partir de estos principios, la CPE pretendió regular la competencia que la misma Constitución otorgó al Ejecutivo en estados de anormalidad institucional, y convirtió tales facultades en actuaciones limitadas y regladas.

En términos generales, los principios que rigen el estado de excepción se pueden definir de la siguiente manera:

- El principio de necesidad alude a la ausencia de mecanismos ordinarios para superar las situaciones que impone la anormalidad. En otros términos, este principio implica que solo en la medida en que las instituciones jurídicas ordinarias sean insuficientes para superar la emergencia, es procedente declarar el estado de excepción.

- El principio de proporcionalidad, tal como ha sido analizado por la Corte Constitucional del Ecuador, impone el deber de examinar si la declaratoria del estado de excepción se encuentra en una relación de adecuación con las medidas adoptadas en desarrollo de este. Es decir, por medio del principio de proporcionalidad se pretende proscribir la adopción de medidas arbitrarias, de tal forma que excedan, incluso, la situación que dio origen a la declaración misma del estado de excepción.

- El principio de legalidad reafirma el carácter reglado de los estados de excepción. Por tanto, aunque es cierto que en virtud de la anormalidad institucional el presidente de la República tiene facultades excepcionales para adoptar las medidas necesarias para enervar la crisis, también lo es que en ejercicio de dichas facultades no puede desconocer, prima facie, el ordenamiento jurídico vigente.

- El principio de temporalidad se refiere a la vigencia del estado de excepción, el cual, por expresa disposición constitucional, es de sesenta días, prorrogables hasta por treinta días más, si las causas que motivaron la declaratoria del estado de excepción persisten.

- El principio de territorialidad hace referencia al espacio geográfico en el que regirá el estado de excepción. En los términos de la CPE, el presidente de la República podrá decretar el estado de excepción en todo el territorio nacional o en parte de él. 
- El principio de razonabilidad implica que ni la declaratoria del estado de excepción ni las medidas adoptadas en desarrollo este pueden ser arbitrarias. Por tanto, este principio constituye un mandato de buen juicio que solo se satisface en la medida en que los principios referidos con anterioridad se cumplan.

Vistos los principios que rigen el estado de excepción, es importante precisar que, de acuerdo con la CPE, la declaratoria de este deberá contener la determinación de la causal que lo decretó y, por supuesto, la correspondiente motivación. Esto implica precisar, de un lado, el ámbito territorial de aplicación, el periodo de duración, las medidas que deberán aplicarse, los derechos que podrán suspenderse o limitarse y, del otro, las notificaciones que correspondan de acuerdo con la Constitución y los tratados internacionales.

Sobre este último particular, vale la pena señalar que la regulación constitucional del estado de excepción en Ecuador dispone que, en contextos de excepción, el presidente de la República únicamente podrá suspender o limitar el ejercicio del derecho a la inviolabilidad de domicilio, inviolabilidad de correspondencia, libertad de tránsito, libertad de asociación y libertad de información ${ }^{2}$.

Además, una vez declarado el estado de excepción, el presidente estará habilitado para decretar, entre otras medidas, i) la recaudación anticipada de tributos, ii) utilizar los fondos públicos destinados a otros fines, excepto los correspondientes a salud y educación y iii) disponer el cierre o la habilitación de puertos, aeropuertos y pasos fronterizos y iv) disponer la movilización y las requisiciones que sean necesarias, y decretar la desmovilización nacional, cuando se restablezca la normalidad.

\section{A. Control constitucional de los estados de excepción en Ecuador}

En Ecuador, la Corte Constitucional es el máximo órgano de control, interpretación constitucional y de administración de justicia en esta materia. Por tanto, a esta corporación le corresponde efectuar de oficio y de modo inmediato el control de constitucionalidad de las declaratorias de los estados de excepción, cuando quiera que estos impliquen la suspensión de derechos constitucionales ${ }^{3}$. Según la CPE, las sentencias y los autos de la Corte Constitucional en esta materia tendrán el carácter de definitivos e inapelables ${ }^{4}$.

Sobre este particular conviene destacar que, aunque por expresa disposición constitucional, el control judicial de las declaratorias de los estados de excepción opera cuando estos impliquen la suspensión de derechos constitucionales, lo cierto es que a partir de la jurisprudencia de la Corte Constitucional se ha considerado que la Corte también se debe pronunciar respecto de la constitucionalidad de todos los decretos de estados de

Constitución Política del Ecuador, art. 165.

3 Constitución Política del Ecuador, art. 438, núm. 8.

4 Constitución Política del Ecuador, art. 440. 
excepción ${ }^{5}$. Dicho de otra manera, toda declaratoria de estado de excepción implica, per se, un control automático de constitucionalidad por parte de la Corte Constitucional.

En tales términos, los artículos 166 de la CPE y 124 de la Ley Orgánica de Garantías Jurisdiccionales y Control Constitucional (LOGJC), prevén expresamente que una vez que el presidente de la República ha decretado el estado de excepción, en los términos previamente señalados, deberá notificar a la Asamblea Nacional, a la Corte Constitucional y a los organismos internacionales que corresponda ${ }^{6}$, dentro de las 48 horas siguientes a la firma del decreto correspondiente.

A partir de las anteriores consideraciones, corresponde a la Corte Constitucional ejercer el control judicial de los estados de excepción.

\section{B. Alcance del control constitucional de los estados de excepción}

La regulación constitucional y orgánica de los estados de excepción prevé que el control constitucional que ejerce la Corte tiene por objeto garantizar el disfrute pleno de los derechos constitucionales y salvaguardar los principios de separación y equilibrio de los poderes públicos. Para ese propósito, la referida LOGJC dispone expresamente que la Corte Constitucional deberá efectuar un control formal y material de los decretos que declaren un estado de excepción y de los decretos que se dicten con fundamento y en desarrollo en este ${ }^{7}$.

\section{CONTROL FORMAL}

El control formal de los estados de excepción procede, por un lado, respecto de la declaratoria de estado de excepción que efectúa el presidente de la República y, por el otro, respecto de las medidas que este expide con fundamento y en desarrollo de la declaratoria del estado de excepción. Esta doble dimensión del control formal se explica en los mandatos de los artículos 120 y 122 de la LOGJC.

De acuerdo con el artículo 120 de la LOGJC, corresponde a la Corte Constitucional verificar que el decreto de la declaratoria del estado de excepción cumpla con los siguientes requisitos: i) identificación de los hechos y de la causal constitucional que se invoca; ii) justificación de la declaratoria; iii) ámbito territorial y temporal de la declaratoria; iv) derechos que sean susceptibles de limitación, cuando fuere el caso; y, v) las notificaciones que correspondan de acuerdo a la Constitución y a los tratados internacionales.

5 Corte Constitucional del Ecuador, Sentencia 0002-09-SEE-CC.

6 El control político es facultativo de la Asamblea Nacional, mientras que el control constitucional corresponde a la Corte Constitucional. Además, dentro del control jurídico es importante destacar el control "externo" a cargo de los organismos internacionales (i. e. la Comisión Interamericana de Derechos Humanos - CIDH). 
Según el artículo 122 de la LOGJC, a la Corte Constitucional le corresponde verificar, además, que las medidas adoptadas con fundamento en la declaratoria de estado de excepción cumplan al menos los siguientes requisitos formales: i) que se ordenen mediante decreto, de acuerdo con las formalidades que establece el sistema jurídico; ii) que se enmarquen dentro de las competencias materiales, espaciales y temporales de los estados de excepción.

Para la Corte Constitucional del Ecuador, esta distinción del examen formal carece de sustancialidad. Esto es así porque, en la práctica, la Corte ha optado por analizar, en conjunto, la totalidad de los requisitos previstos para adelantar el control formal del decreto por medio del cual se declara el estado de excepción y los decretos expedidos con fundamento y en desarrollo de dicha declaratoria.

\section{CONTROL MATERIAL}

El control material de constitucionalidad, así como el control formal, implican, de un lado, el análisis del decreto por medio del cual el presidente de la República declara el estado de excepción y, del otro, el examen de los decretos expedidos con fundamento y en desarrollo de la declaratoria del estado de excepción. Esto es así, en atención a los artículos 121 y 123 de la LOGJC.

Por un lado, en relación con el control material de la declaratoria del estado de excepción, el artículo 121 de la LOGJC dispone que a la Corte Constitucional le corresponde verificar, al menos, lo siguiente: i) que los hechos alegados en la motivación hayan tenido real ocurrencia; ii) que los hechos constitutivos de la declaratoria configuren una agresión, un conflicto armado internacional o interno, grave conmoción interna, calamidad pública o desastre natural; iii) que los hechos constitutivos de la declaratoria no puedan ser superados por medio del régimen constitucional ordinario; iv) que la declaratoria se decrete dentro de los límites temporales y espaciales establecidos en la Constitución.

Por otro lado, según el artículo 123 de la LOGJC, en relación con las medidas adoptadas con fundamento y en desarrollo del estado de excepción, corresponde a la Corte examinar que dichas medidas: i) sean estrictamente necesarias para enfrentar los hechos que dieron lugar a la declaratoria; ii) sean proporcionales al hecho que dio lugar a la declaratoria; iii) exista una relación de causalidad directa e inmediata entre los hechos que dieron lugar a la declaratoria y las medidas adoptadas; iv) sean idóneas para enfrentar los hechos que dieron lugar a la declaratoria; v) no exista otra medida que genere un menor impacto en términos de derechos y garantías; vi) no afecten el núcleo esencial de los derechos constitucionales, y se respeten el conjunto de derechos intangibles; y, vii) no se interrumpa ni se altere el normal funcionamiento del Estado.

De conformidad con lo anterior, es plausible concluir que el control material de constitucionalidad de la declaratoria del estado de excepción y las medidas expedidas en desarrollo este, tienen por objeto verificar el cumplimiento de los principios que rigen los 
estados de excepción en los términos del artículo 164 de la CPE. Es decir, los principios de necesidad, proporcionalidad, legalidad, temporalidad, territorialidad y razonabilidad.

Así, por ejemplo, en la Sentencia 0001-09-SEE-CC, la Corte Constitucional del Ecuador considero,

... d) Las medidas a adoptarse durante el estado de excepción, son adecuadas al fin que se pretende conseguir, cumpliendo así el principio de proporcionalidad que supone la existencia de un peligro inminente y exige una adecuación entre éste y los medios utilizados para repelerlo; a su vez, estos para ser legítimos deben ser proporcionales a la gravedad del peligro. Frente a los hechos generadores de la situación de crisis en el sistema Petroecuador las medidas enunciadas en el Decreto Ejecutivo no son desproporcionadas si se considera el peligro que correría el Estado y la sociedad en caso de implementarlas.

\section{La institución del estado de excepción en la Constitución Política de Colombia}

La Constitución Política de Colombia (CPC) prevé que el presidente de la República podrá declarar tres tipos de estado de excepción, a saber: i) guerra exterior, ii) conmoción interior y iii) emergencia económica, social y ecológica. Esta distinción obedece a la naturaleza de la crisis o la emergencia.

En primer lugar, el artículo 212 de la CPC dispone que el presidente de la República, con la firma de todos los ministros, podrá declarar el estado de guerra exterior. Mediante tal declaración, el Gobierno tendrá las facultades estrictamente necesarias para repeler la agresión, defender la soberanía, atender los requerimientos de la guerra y procurar el restablecimiento de la normalidad.

En segundo lugar, el artículo 213 de la CPC prevé expresamente que en caso de grave perturbación del orden público que atente de manera inminente contra la estabilidad institucional, la seguridad del Estado o la convivencia ciudadana, y que no pueda ser conjurada mediante el uso de las atribuciones ordinarias de las autoridades de Policía, el presidente de la República, con la firma de todos los ministros, podrá declarar el Estado de conmoción interior en toda la República o parte de ella, por un término no mayor de noventa días, prorrogable hasta por dos periodos iguales, el segundo de los cuales requiere concepto previo y favorable del Senado de la República.

En tercer lugar, el artículo 215 de la CPC prevé que el presidente de la República podrá declarar el estado de emergencia cuando sobrevengan hechos distintos de los previstos como constitutivos del estado de guerra exterior o del estado de conmoción interior (arts. 212 y 213 de la CPC), y configuren una perturbación, o amenaza de perturbación, de los órdenes social, económico o ecológico del país, por periodos hasta de treinta días en cada caso, que sumados no podrán exceder de noventa días en el año calendario. 
De acuerdo con la jurisprudencia constitucional ${ }^{8}$, los tres tipos de estados de excepción tienen un carácter reglado, excepcional y limitado. En efecto, según el artículo 214 de la CPC, los estados de excepción a que se refieren los citados artículos se someterán a las siguientes disposiciones:

i. Los decretos legislativos llevarán la firma del presidente de la República y todos sus ministros y solamente podrán referirse a materias que tengan relación directa y específica con la situación que hubiere determinado la declaratoria del estado de excepción.

ii. Los decretos legislativos no podrán suspender los derechos humanos ni las libertades fundamentales. En todo caso, se respetarán las reglas del derecho internacional humanitario (DIH) y las medidas que se adopten deberán ser proporcionales a la gravedad de los hechos.

iii. No se interrumpirá el normal funcionamiento de las ramas del poder público ni de los órganos del Estado.

iv. Tan pronto como hayan cesado la guerra exterior o las causas que dieron lugar al estado de conmoción interior, el Gobierno declarará restablecido el orden público y levantará el estado de excepción.

\section{A. Control constitucional de los estados de excepción en Colombia}

El carácter reglado, excepcional y limitado de los estados de excepción implica, entre otras medidas, un control constitucional de los decretos legislativos que dicte el presidente de la República en uso de las facultades a que se refieren los artículos 212, 213 y 215 de la CPC previamente referidos.

En los términos del artículo 214 de la cPC, el Gobierno enviará a la Corte Constitucional los decretos legislativos que dicte en uso de las facultades a que se refieren los artículos 212, 213 y 215 de la CPC, a fin de que esta decida definitivamente sobre su constitucionalidad. Si el Gobierno no cumpliere con el deber de enviarlos, la Corte Constitucional aprehenderá de oficio y en forma inmediata su conocimiento.

De esto se sigue el Gobierno nacional hubiese remitido, al día siguiente de su declaratoria, el Decreto Legislativo 417 de 2020, y que la totalidad de los decretos legislativos expedidos con fundamento y desarrollo de este hayan sido remitidos a la Corte Constitucional para el correspondiente control judicial. 


\section{B. Alcance del control constitucional de los estados de excepción}

En los términos del artículo 241 de la CPC, a la Corte Constitucional le corresponde decidir definitivamente sobre la constitucionalidad de los decretos legislativos que dicte el Gobierno nacional con fundamento en los artículos 212, 213 y 215 de la Constitución. Esto implica, de un lado, el control constitucional de la declaratoria del estado de excepción y, del otro, el control constitucional de las medidas adoptadas con fundamento y en desarrollo de dicha declaratoria.

En los términos expuestos por la jurisprudencia constitucional en la Sentencia C-400 de 2013,

... la función de adelantar el control abstracto de constitucionalidad reposa principalmente en la Corte Constitucional, instituida como el órgano principal al que "se le confía la guarda de la integridad y supremacía de la Constitución", labor que debe ser desarrollada en "los estrictos y precisos términos" del artículo 241 superior, el cual prescribe de manera taxativa las funciones que le competen.

De esta manera, la Corte Constitucional tiene la más amplia competencia sobre el control abstracto de constitucionalidad.

Ahora bien, de acuerdo con la misma jurisprudencia constitucional, el control constitucional de los decretos expedidos con fundamento y en desarrollo de los estados de excepción tiene dos facetas ${ }^{9}$ : una formal y otra material. Para la Corte Constitucional, esta doble dimensión del control judicial se fundamenta en un escrutinio detallado que tiene por objeto asegurar que todos los límites previstos para el ejercicio de las facultades de excepción sean debidamente respetados por el presidente de la República.

\section{CONTROL FORMAL}

El control formal de los estados de excepción exige verificar si los decretos legislativos expedidos por el presidente de la República: i) están suscritos por el presidente y todos los ministros, ii) fueron expedidos durante la vigencia y en desarrollo del respectivo estado de excepción, iii) están motivados, iv) establecen claramente su duración, v) fueron remitidos a la Corte Constitucional al día siguiente de su adopción para la revisión automática de constitucionalidad y, finalmente, vi) determinan el ámbito territorial para su aplicación ${ }^{10}$.

9 Cfr. sentencias C-466 de 2017, C-722 de 2015, C-300 de 2011, C-244 de 2011, C-233 de 2011, C-216 de 2011 , C-194 de 2011, C-193 de 2011 y C-940 de 2002.

Idem. 
$\mathrm{Al}$ respecto, conviene precisar que el control formal de constitucionalidad procede respecto de la declaratoria del estado de excepción y, además, respecto de los decretos legislativos expedidos con fundamento y en desarrollo de dicha declaratoria.

\section{Control MATERIAL}

El control material se compone de varios escrutinios que constituyen expresiones operativas de los principios que rigen los estados de excepción. En particular, el examen material de la declaratoria del estado de excepción y de los decretos legislativos expedidos con fundamento y en desarrollo de dicha declaratoria se ha estructurado a partir de diez juicios (escrutinios) materiales elaborados por la Corte Constitucional, habida cuenta de las disposiciones constitucionales y estatutarias que rigen la materia, a saber: i) juicio de finalidad, ii) juicio de conexidad material, iii) juicio de motivación suficiente, iv) juicio de ausencia de arbitrariedad, v) juicio de intangibilidad, vi) juicio de no contradicción específica, vii) juicio de incompatibilidad, viii) juicio de necesidad, ix) juicio de proporcionalidad y x) juicio de no discriminación.

En particular, cada uno de estos juicios se puede explicar de la siguiente manera:

- El juicio de finalidad está previsto en el artículo 10 de la Ley Estatutaria de Estados de Excepción (LEEE). Este juicio dispone que las medidas adoptadas por los decretos legislativos deberán estar directa y específicamente encaminadas a enervar las causas de la perturbación y a impedir la extensión de sus efectos.

- El juicio de conexidad material está previsto por el artículo 47 de la LEEE. De acuerdo con esta disposición, en virtud de la declaración del estado de excepción, el Gobierno podrá dictar decretos con fuerza de ley, destinados exclusivamente a conjurar la crisis y a impedir la extensión de sus efectos ${ }^{11}$.

- El juicio de motivación suficiente está previsto en el artículo 8 de la LEEE. Este juicio ha sido entendido como complementario al control formal de constitucionalidad, por cuanto tiene por objeto determinar si el presidente de la República presentó razones suficientes para justificar las medidas adoptadas en desarrollo del estado de excepción ${ }^{12}$. En tales términos, los decretos de excepción deberán señalar los motivos por los cuales se imponen cada una de las limitaciones de los derechos constitucionales.

- El juicio de ausencia de arbitrariedad tiene por objeto comprobar que en el decreto legislativo no se establezcan medidas que violen las prohibiciones para el ejercicio

11 Tradicionalmente, la Corte Constitucional ha examinado la conexidad material de las medidas de excepción a partir de dos dimensiones: i) interna y ii) externa. La primera, hace referencia a la relación entre las medidas adoptadas y las consideraciones expresadas por el Gobierno nacional para motivar el decreto de desarrollo correspondiente. La segunda, se refiere al vínculo entre las medidas de excepción y los motivos que dieron lugar a la declaratoria del estado de emergencia. Cfr. Sentencias C-724 de 2015 y C-701 de 2015. 
de las facultades extraordinarias reconocidas en la Constitución, la LEEE y los tratados internacionales de derechos humanos ratificados por Colombia ${ }^{13}$.

- El juicio de intangibilidad prevé que algunos de los derechos previstos por los artículos 93 y 214 de la $\mathrm{CPC}^{14}$ no pueden ser limitados o restringidos ni siquiera durante los estados de excepción.

- El juicio de no contradicción específica implica verificar que las medidas adoptadas en los decretos legislativos no contraríen de manera concreta la Constitución, los tratados internacionales y, en general, el marco de referencia de la actuación del Ejecutivo en el estado de excepción. Esto es, el grupo de medidas descritas en los artículos 47, 49 y 50 de la LEEE.

- El juicio de incompatibilidad esta previsto por el artículo 12 de la LEEe. Según esta disposición, los decretos legislativos que suspendan leyes deben expresar las razones por las cuales son irreconciliables con el correspondiente estado de excepción.

- El juicio de necesidad está previsto en el artículo 11 de la LEEE. Este juicio tiene por objeto determinar si las medidas adoptadas son indispensables para lograr los fines que dieron lugar a la declaratoria del estado de excepción ${ }^{15}$.

- El juicio de proporcionalidad está previsto en el artículo 13 de la LEEE. Este juicio precisa, de un lado, que las medidas expedidas durante los estados de excepción deberán guardar proporcionalidad con la gravedad de los hechos que buscan conjurar y, del otro, que la limitación en el ejercicio de los derechos y las libertades solo será admisible en el grado estrictamente necesario, para buscar el retorno a la normalidad.

- El juicio de no discriminación, previsto en el artículo 14 de la LEEe, implica verificar que las medidas adoptadas con ocasión de los estados de excepción no pueden entrañar discriminación alguna fundada en razones de raza, lengua, religión, origen nacional o familiar, opinión política o filosófica, o de otras categorías sospechosas.

13 Sentencias C-466 de 2017, C-723 de 2015 y C-742 de 2015.

14 La Corte ha establecido que en virtud del derecho internacional de los derechos humanos, se consideran como intangibles el derecho a la vida y a la integridad personal; el derecho a no ser sometido a desaparición forzada, a torturas, ni a tratos o penas crueles, inhumanos o degradantes; el derecho al reconocimiento de la personalidad jurídica; la prohibición de la esclavitud, la servidumbre y la trata de seres humanos; la prohibición de las penas de destierro, prisión perpetua y confiscación; la libertad de conciencia; la libertad de religión; los principios de legalidad, de favorabilidad y de irretroactividad de la ley penal; el derecho a elegir y ser elegido; los derechos a contraer matrimonio y a la protección de la familia; el derecho del niño a la protección por parte de su familia, de la sociedad y del Estado; el derecho a no ser condenado a prisión por deudas, y el derecho al habeas corpus. Son igualmente intangibles los mecanismos judiciales indispensables para la protección de esos derechos.

$15 \mathrm{Al}$ analizar este juicio, la Corte Constitucional ha evaluado: i) si fácticamente las medidas adoptadas permiten superar la crisis o evitar la extensión de sus efectos (necesidad fáctica), y ii) si en el ordenamiento jurídico no existían previsiones ordinarias que fueran suficientes y adecuadas para lograr los objetivos de la medida excepcional (necesidad jurídica). 


\section{Conclusiones}

La dificultad que entraña el uso indiscriminado de los estados de excepción como instrumento de política gubernamental determinó la regulación y limitación de esta institución. En este sentido, por ejemplo, la CPE prevé que la declaratoria del estado de excepción se regirá, en todos los casos, por los principios de necesidad, proporcionalidad, legalidad, temporalidad, territorialidad y razonabilidad. Lo propio sucede con la CPC que dispone expresamente que los decretos legislativos expedidos en el marco de los estados de excepción deberán ser proporcionales a la gravedad de los hechos que lo suscitaron.

En términos generales, el control constitucional que ejercen los Tribunales constitucionales del Ecuador y Colombia respecto de los estados de excepción es automático y de oficio. Una vez decretado el estado de excepción -por cualquiera de sus causas-, el presidente de la República debe remitir a la Corte Constitucional el decreto correspondiente para su respectivo control judicial. De no hacerlo, tanto la Corte Constitucional del Ecuador como la Corte Constitucional de Colombia están expresamente habilitadas para conocer de oficio este decreto. Esta circunstancia denota el carácter reglado y limitado de los estados de excepción en ambos países. Esto es así, entre otras razones, por el uso habitual e indiscriminado de esta institución en detrimento de la democracia y la vigencia integral de los derechos fundamentales de los ciudadanos.

Una de las notas características del control constitucional que ejercen estos tribunales es que procede, de un lado, respecto del decreto por medio del cual el presidente de la República declara el estado de excepción y, por tanto, asume las facultades extraordinarias conferidas constitucionalmente en este tipo de contextos, y, del otro, los decretos -legislativos en el caso de Colombia- que expide en desarrollo de dicho decreto que declaró el estado de excepción.

Adicionalmente, es importante comentar que el control constitucional que ejercen estos tribunales es formal y material. Así, por ejemplo, la regulación constitucional y orgánica de Ecuador prevé que por medio del control formal de la declaratoria del estado de excepción y las medidas adoptadas con fundamento en su declaratoria, la Corte deberá verificar el cumplimiento de los siguientes requisitos: i) identificación de los hechos y de la causal constitucional que se invoca; ii) justificación de la declaratoria; iii) ámbito territorial y temporal de la declaratoria; iv) derechos que sean susceptibles de limitación, cuando fuere el caso; v) las notificaciones que correspondan de acuerdo con la Constitución y los tratados internacionales, vi) que se ordenen mediante decreto, de acuerdo con las formalidades que establece el sistema jurídico; y, vii) que se enmarquen dentro de las competencias materiales, espaciales y temporales de los estados de excepción.

De otro lado, por medio del control material, la Corte deberá verificar que: i) los hechos alegados en la motivación hayan tenido real ocurrencia; ii) los hechos constitutivos de la declaratoria configuren una agresión, un conflicto armado internacional o interno, grave conmoción interna, calamidad pública o desastre natural; iii) los hechos constitutivos de la declaratoria no puedan ser superados por medio del régimen constitucional 
ordinario; iv) la declaratoria de excepción se decrete dentro de los límites temporales y espaciales establecidos en la Constitución; v) las medidas sean estrictamente necesarias para enfrentar los hechos que dieron lugar a la declaratoria, y las medidas ordinarias sean insuficientes para el logro de este objetivo; vi) sean proporcionales al hecho que dio lugar a la declaratoria; vii) tengan relación de causalidad directa e inmediata entre los hechos que dieron lugar a la declaratoria; viii) sean idóneas para enfrentar los hechos que dieron lugar a la declaratoria; ix) no exista otra medida que genere un menor impacto en términos de derechos y garantías; x) no afecten el núcleo esencial de los derechos constitucionales, $\mathrm{y}$ se respete el conjunto de derechos intangibles; $\mathrm{y}$, xi) no interrumpan ni alteren el normal funcionamiento del Estado.

En el caso colombiano, por su parte, a la Corte Constitucional le corresponde determinar, a partir de un examen formal, si los decretos legislativos expedidos en el marco de los estados de excepción: i) están suscritos por el presidente de la República y todos los ministros, ii) han sido expedidos durante la vigencia y en desarrollo del respectivo estado de excepción, iii) están motivados, iv) establecen claramente su duración, v) fueron remitidos a la Corte Constitucional, al día siguiente de su adopción, para la revisión automática de constitucionalidad, y, finalmente, vi) determinan el ámbito territorial para su aplicación.

Adicionalmente, a partir de un examen material, le corresponde determinar si superan los diez juicios materiales (escrutinios) elaborados, a saber: i) juicio de finalidad, ii) juicio de conexidad material, iii) juicio de motivación suficiente, iv) juicio de ausencia de arbitrariedad, v) juicio de intangibilidad, vi) juicio de no contradicción específica, vii) juicio de incompatibilidad, viii) juicio de necesidad, ix) juicio de proporcionalidad y x) juicio de no discriminación.

\section{Referencias}

Cifuentes Muñoz, E. (2002). Los estados de excepción constitucional en Colombia. Ius et Praxis, 8(1), 117-146.

García Villegas, M. y Uprimny, R. ¿Controlando la excepcionalidad permanente en Colombia? Una defensa prudente del control judicial de los estados de excepción. Documentos de Discusión de Dejusticia, (1). www.dejusticia.org.

Gargarella, R. y Roa Roa, J. (2020). Democratic dialogue and emergency in Latin America. Max Planck Institute for Comparative Public Law \& International Law (MPIL), Research Paper 2020-21.

Oficina del Alto Comisionado de las Naciones Unidas para los Derechos Humanos (ACNUDH) (2020). Las medidas de emergencia y el Covid-19: orientaciones, 27 de abril de 2020. ONu. https:/www.ohchr.org/Documents/Events/ EmergencyMeasures_COVID19_ES.pdf 
Organización Mundial de la Salud (OMs) (2020). actualización de la estrategia frente a la covid-19. OMS.

Restrepo Piedrahíta, C. (1988). El síndrome del presidencialismo en Colombia. Universidad Externado de Colombia.

Vanegas Gil, P. La Constitución colombiana y los estados de excepción: veinte años después. Revista Derecho del Estado, 27 (1), 261-290.

\section{Normativa}

Ley 137 de 2 de junio de 1994, "Por la cual se reglamentan los Estados de Excepción en Colombia".

Ley Orgánica de Garantías Jurisdiccionales y Control Constitucional, de 10 de septiembre de 2009.

Presidencia de la República de Colombia (2020). Decreto Ejecutivo 1017 de 16 de marzo de 2020 .

Presidencia de la República de Colombia (2020). Decreto Legislativo 417 de 17 de marzo de 2020 .

Presidencia de la República de Colombia (1991). Constitución Política de Colombia.

Presidencia de la República de Ecuador (2008). Constitución Política del Ecuador.

\section{Jurisprudencia:}

Corte Constitucional de Colombia, Sentencia C-193 de 2011.

Corte Constitucional de Colombia, Sentencia C-194 de 2011.

Corte Constitucional de Colombia, Sentencia C-216 de 2011.

Corte Constitucional de Colombia, Sentencia C-233 de 2011.

Corte Constitucional de Colombia, Sentencia C-244 de 2011.

Corte Constitucional de Colombia, Sentencia C-300 de 2011.

Corte Constitucional de Colombia, Sentencia C-400 de 2013.

Corte Constitucional de Colombia, Sentencia C-466 de 2017.

Corte Constitucional de Colombia, Sentencia C-701 de 2015

Corte Constitucional de Colombia, Sentencia C-722 de 2015. 
Corte Constitucional de Colombia, Sentencia C-723 de 2015

Corte Constitucional de Colombia, Sentencia C-724 de 2015

Corte Constitucional de Colombia, Sentencia C-742 de 2015

Corte Constitucional de Colombia, Sentencia C-940 de 2002.

Corte Constitucional del Ecuador, Dictamen No. 2-20-EE/20.

Corte Constitucional del Ecuador, Sentencia 0001-09-SEE-CC.

Corte Constitucional del Ecuador, Sentencia 0002-09-SEE-CC.

Corte Constitucional del Ecuador, Sentencia 0017-10-SEE-CC.

Fecha de recepción: 17 de junio de 2020

Fecha de aprobación par 1: 04 de julio de 2020

Fecha de aprobación par 2: 14 de julio de 2020 